\title{
Accelerated Degradation Process Analysis Based on the Nonlinear Wiener Process with Covariates and Random Effects
}

\author{
Li Sun, ${ }^{1}$ Xiaohui Gu, ${ }^{1}$ and Pu Song ${ }^{2}$ \\ ${ }^{1}$ Nanjing University of Science and Technology, Nanjing, Jiangsu 210094, China \\ ${ }^{2}$ Science and Technology on Combustion and Explosion Laboratory, Xian, Shanxi 710065, China
}

Correspondence should be addressed to Xiaohui Gu; gxiaohui@njust.edu.cn

Received 13 September 2016; Accepted 27 November 2016

Academic Editor: Eusebio Valero

Copyright (c) 2016 Li Sun et al. This is an open access article distributed under the Creative Commons Attribution License, which permits unrestricted use, distribution, and reproduction in any medium, provided the original work is properly cited.

It is assumed that the drift parameter is dependent on the acceleration variables and the diffusion coefficient remains the same across the whole accelerated degradation test (ADT) in most of the literature based on Wiener process. However, the diffusion coefficient variation would also become obvious in some applications with the stress increasing. Aiming at the phenomenon, the paper concludes that both the drift parameter and the diffusion parameter depend on stress variables based on the invariance principle of failure mechanism and Nelson assumption. Accordingly, constant stress accelerated degradation process (CSADP) and step stress accelerated degradation process (SSADP) with random effects are modeled. The unknown parameters in the established model are estimated based on the property of degradation and degradation increment, separately for CASDT and SSADT, by the maximum likelihood estimation approach with measurement error. In addition, the simulation steps of accelerated degradation data are provided and simulated step stress accelerated degradation data is designed to validate the proposed model compared to other models. Finally, a case study of CSADT is conducted to demonstrate the benefits of our model in the practical engineering.

\section{Introduction}

For many highly reliable products, it is not an easy task to obtain their life information by using traditional life test because failures are not likely to occur in a certain period of time, even by censoring life test and accelerated life test. In such a case, degradation data which is related to life is used due to the following reasons: ease of obtaining, low cost, short test period, and informative data. And it has been widely used in classification [1], residual life estimation [2], reliability assessment [3], and so on. To model the degradation data, two classes of models have been well exploited, general path model and stochastic process model $[4,5]$. The general path model is first introduced by $\mathrm{Lu}$ and Meeker in 1993 [6] whose failure time is determined with known random parameters. But it may not be good at describing the inherent randomness of each product and the unexplained randomness and dynamics due to unobserved environmental factors. There are various stochastic process models, including Wiener process [7], Gamma process [8], Geometric Brownian Motion Process [9], and Inverse Gaussian
Process $[10,11]$. Beyond all of the stochastic process models, Wiener process has been used intensively for its flexible and meaningful characteristic. In addition, Wiener process has more advantages than other stochastic process models for nonmonotonic degradation data.

Most of the degradation data mentioned above is degradation data under normal stress or field degradation data. However, the life information should be obtained in a shorter period of time for some products, especially for newly developed products and highly reliable components. Instead, it is a lengthy and drawn-out process to collect field degradation data. Under the circumstances, ADT is a suitable choice to gather the life information quickly and efficiently.

In general, with more accelerated degradation data and higher measuring precision, we can achieve higher accuracy for forecasting parameters, but the experiment cost would increase correspondingly. So we can deal with the optimal accelerated degradation plan (including the optimal settings for the sample size, accelerated stresses, measurement frequency, and termination time) for a Wiener degradation process by minimizing the approximate variance of the 
estimated mean time to failure under the constraint that the total experimental cost does not exceed a prespecified budget or minimizing the testing cost under the condition of a maximum acceptable approximate standard error. Some well-known references on the optimization of CSADT based on Wiener process are Lim and Yum [5] and Tsai et al. [12]. The optimization of SSADT based on Wiener process can be referred to in the research of Liao and Tseng [13], Tang et al. [14], and $\mathrm{Hu}$ et al. [15]. In addition, accelerated degradation model is another hot area which has attracted much attention of the researchers. Liao and Tseng [13] modeled the step stress accelerated degradation data of LED lamps. Considering unit-to-unit variability, a step stress accelerated degradation model based on the basic Wiener process was proposed by Tang [16]. However, it is often found that the degradation path is not always linear. So an accelerated degradation process modeling method with random effects for the nonlinear Wiener process was established by Tang et al. [17] later. Wang et al. [18] proposed a Bayesian evaluation method to integrate the ADT data from laboratory with the failure data from field.

The above literature all assumed that the drift parameter is dependent on the acceleration variables and the diffusion coefficient remains the same across the whole ADT. But when the degradation rate increases, the degradation variation would also become larger in some applications [19]. WHITMORE [20] fitted the degradation data of each product separately with a time scale transformed Wiener process and then the parameter transformation was tentatively identified based on the plots against the reciprocal of the absolute temperature. The plots revealed that both the drift parameter and the diffusion parameter of self-regulating heating cable are increasing with the increment of temperature. Doksum and Hoyland [21] introduced the conception of the multiplicative factor, and it was assumed that the drift parameter and diffusion parameter are multiples of the multiplicative factor whose expression with accelerated stress level provides a good model fit of one of the four empirical models. Liao and Elsayed [22] extended an accelerated degradation model to predict field reliability by considering the stress variations where the drift parameter and the diffusion parameter are expressed by the different function of the constant stress vector. Ye [19] proposed a new random effects Wiener process model such that the drift parameter is a particular multiple of the diffusion parameter and the unknown parameters were calculated by EM algorithm. The relationship between the drift parameter and diffusion parameter was either an assumption or just the fitting according to specific test data.

The paper is motivated by the latest paper of Wang et al. [23] in which they deduced that the ratio of drift parameters under two different stresses is equal to the acceleration factor, as well as the ratio of diffusion parameters. Based on this conclusion, we model the constant stress accelerated degradation process (CSADP) and the step stress accelerated degradation process (SSADP) in consideration of random effects. Moreover, the unknown parameters in the model, including measurement error, are obtained by using the maximum likelihood estimation (MLE) method. Besides, a numerical example and a case study are presented to verify the superiority of the model proposed in this paper compared with other two models.

The remainder of this paper is organized as follows: Section 2 develops the nonlinear Wiener process and deduces the relationships of parameters in ADT and the probability density function (PDF) and cumulative distribution function (CDF) under a certain stress with random effects. Section 3 models the degradation process in CSADT and SSADT. Section 4 describes the procedure for parameter estimation for two cases. Two numerical examples and a practical example are presented to verify the proposed model in Sections 5 and 6 , separately. Section 7 concludes the paper with a discussion.

\section{Nonlinear Wiener Process with Covariates and Random Effects}

2.1. The Wiener Process with Time Scale Transformation. The time-transformed Wiener process is commonly used to model the nonlinear accelerated degradation data [17]. Let $X(t)$ denote the degradation value at time $t$; then the Wiener degradation process with time scale transformation can be represented as follows [20]:

$$
X(t)=\lambda \Lambda(t)+\sigma_{B} B(\Lambda(t))
$$

where $\lambda$ is the drift parameter, $\sigma_{B}$ is the diffusion parameter, $t$ denotes the clock or calendar time, and $\Lambda(t)$ is the transformed time whose selection can be referred to in Section 6 of literature [24]. $B(\Lambda(t))$ is the standard Brownian motion which represents the stochastic dynamics of the degradation process at transformed time scale. If $\Lambda(t)=t$, the nonlinear Wiener process becomes the traditional Wiener process [17]. Generally, if $X(t)$ reaches a specific value $w$ which is related to the failure mechanism in most cases for the first time, the product is announced to be failed and the time is thus called the first hitting time (FHT). Given $w, \lambda$, and $\sigma_{B}$, it is known that the transformed FHT in such a case follows an inverse Gaussian distribution [11], with corresponding PDF and CDF as

$f(t)$

$$
=\frac{w}{\sqrt{2 \pi \sigma_{B}^{2}(\Lambda(t))^{3}}} \exp \left(-\frac{(w-\lambda \Lambda(t))^{2}}{2 \sigma_{B}^{2} \Lambda(t)}\right) \frac{d \Lambda(t)}{d t},
$$

$F(t)$

$$
\begin{aligned}
& =\Phi\left(\frac{\lambda \Lambda(t)-w}{\sqrt{\sigma_{B}^{2} \Lambda(t)}}\right) \\
& +\exp \left(\frac{2 \lambda w}{\sigma_{B}^{2}}\right) \Phi\left(-\frac{\lambda \Lambda(t)+w}{\sqrt{\sigma_{B}^{2} \Lambda(t)}}\right),
\end{aligned}
$$

where $\Phi(\cdot)$ denotes a standard normal distribution function.

2.2. Deducing the Relationship of Parameters in ADT Based on Nonlinear Wiener Process. ADT is a method to accelerate the 
TABLE 1: Three accelerated models and their acceleration factor.

\begin{tabular}{llll}
\hline Accelerated models & Drift parameter $\lambda$ & Diffusion parameter $\sigma_{B}$ & Acceleration factor \\
\hline Arrhenius model & $\lambda_{k}=\eta \exp \left(-\frac{\beta}{S_{k}}\right)$ & $\left(\sigma_{B}^{2}\right)_{k}=\kappa \exp \left(-\frac{\beta}{S_{k}}\right)$ & $\alpha_{k, 0}=\exp \left(-\beta\left(\frac{1}{S_{k}}-\frac{1}{S_{0}}\right)\right)$ \\
Inverse power model & $\lambda_{k}=\eta \exp \left(-\beta \ln \left(S_{k}\right)\right)$ & $\left(\sigma_{B}^{2}\right)_{k}=\kappa \exp \left(-\beta \ln \left(S_{k}\right)\right)$ & $\alpha_{k, 0}=\exp \left(-\beta\left(\ln \left(S_{k}\right)-\ln \left(S_{0}\right)\right)\right)$ \\
Eyring model & $\lambda_{k}=\frac{\eta}{S_{k} \exp \left(-\beta / S_{k}\right)}$ & $\left(\sigma_{B}^{2}\right)_{k}=\frac{\kappa}{S_{k} \exp \left(-\beta / S_{k}\right)}$ & $\alpha_{k, 0}=\frac{S_{0}}{S_{k} \exp \left(-\beta\left(1 / S_{k}-1 / S_{0}\right)\right)}$ \\
\hline
\end{tabular}

degradation of products by elevating stress, and the obtained degradation data are then used to extrapolate the information through accelerating model to obtain the estimates of life or performance of products at normal use condition. To ensure the accuracy of the extrapolation, the failure mechanism under the accelerated stress and the normal stress must keep the same which is also the premise of the ADT. One of the most common methods for consistency inspection of the failure mechanism is based on statistical method [25]. The principle of this method is that the acceleration factor is a constant and independent of testing time if the failure mechanism remains unchanged. The definition of the acceleration factor is given below according to the Nelson assumption [26].

Specify $\omega=\Lambda(t)$ and suppose $F^{*}$ represents the predetermined cumulative failure probability. $\omega_{0}$ is defined as the testing time when the accumulated failure probability comes to $F^{*}$ under normal stress $S_{0}$, as well as $\omega_{k}$ under accelerated stress $S_{k}$.

$$
F^{*}=F_{k}\left(\omega_{k}\right)=F_{0}\left(\omega_{0}\right) \text {. }
$$

Then the acceleration factor $\alpha_{k, 0}$ of stress $S_{k}$ relative to stress $S_{0}$ can be defined as

$$
\alpha_{k, 0}=\frac{\omega_{0}}{\omega_{k}}
$$

The expression $\omega_{0}=\alpha_{k, 0} \omega_{k}$ can be obtained from (5) and plug it into (4). Then take the first-order derivative with respect to $\omega_{k}$ and we have the following equation for any $\omega_{k}>0$ :

$$
f_{k}\left(\omega_{k}\right)=\alpha_{k, 0} f_{0}\left(\alpha_{k, 0} \omega_{k}\right)
$$

The expression of $f_{k}\left(t_{k}\right)$ and $f_{0}\left(t_{0}\right)$ can be deducted according to (2); then

$$
\begin{aligned}
\alpha_{k, 0} & =\frac{f_{k}\left(\omega_{k}\right)}{f_{0}\left(\alpha_{k, 0} \omega_{k}\right)}=\sqrt{\frac{\left(\alpha_{k, 0}\right)^{3}\left(\sigma_{B}^{2}\right)_{0}}{\left(\sigma_{B}^{2}\right)_{k}}} \\
\cdot & \exp \left[\frac{1}{2 \omega_{k}}\left(\frac{w^{2}}{\left(\sigma_{B}^{2}\right)_{0} \alpha_{k, 0}}-\frac{w^{2}}{\left(\sigma_{B}^{2}\right)_{k}}\right)\right. \\
+ & \left.\frac{\omega_{k}}{2}\left(\frac{\lambda_{0}^{2} \alpha_{k, 0}}{\left(\sigma_{B}^{2}\right)_{0}}-\frac{\lambda_{k}^{2}}{\left(\sigma_{B}^{2}\right)_{k}}\right)+w\left(\frac{\lambda_{k}}{\left(\sigma_{B}^{2}\right)_{k}}-\frac{\lambda_{0}}{\left(\sigma_{B}^{2}\right)_{0}}\right)\right] .
\end{aligned}
$$

The acceleration factor $\alpha_{k, 0}$ is a constant that does not change with $t_{k}$ if and only if the relationship of the parameters is satisfied with

$$
\alpha_{k, 0}=\frac{\lambda_{k}}{\lambda_{0}}=\frac{\left(\sigma_{B}^{2}\right)_{k}}{\left(\sigma_{B}^{2}\right)_{0}} .
$$

Instead of the hypothesis that the diffusion parameter is a constant and does not change with the stress, the conclusion that both the drift parameter $\lambda$ and the diffusion parameter $\sigma_{B}$ depend on stress variables could be drawn based on the previous derivation. At the same time, it was testified that a unit with high realization of the drift parameter would possess a high degradation rate and a high variation in the degradation path in theory which is in conformity with the viewpoint of [19]. The relationship between parameters and accelerated stress variables can be set up by acceleration models based on engineering background. The frequently used acceleration model includes the Arrhenius model, the inverse power model, and the Eyring model whose expressions and acceleration factors are listed in Table 1. Specify $\varsigma\left(S_{k} \mid \beta\right)=\exp \left(-\beta / S_{k}\right), \varsigma\left(S_{k} \mid \beta\right)=\exp \left(-\beta \ln \left(S_{k}\right)\right)$, and $\varsigma\left(S_{k} \mid \beta\right)=\exp \left(-\beta / S_{k}\right) / S_{k}$ to the three models, separately. The accelerated model of drift parameter $\lambda$, the diffusion parameter $\sigma_{B}$, and the accelerated factor $\alpha_{k, 0}$ can be uniformly written as

$$
\begin{aligned}
\lambda_{k} & =\eta \varsigma_{k}, \\
\left(\sigma_{B}^{2}\right)_{k} & =\kappa \varsigma_{k}, \\
\alpha_{k, 0} & =\frac{\varsigma_{k}}{\varsigma_{0}}
\end{aligned}
$$

where $\varsigma_{k}$ is the abbreviated form of $\varsigma\left(S_{k} \mid \beta\right)$ for simplification of the expressions.

2.3. ADT with Random Effects. The observed degradation for products from the same population may be very different owing to unobservable factors $[4,27]$. And there are three sources of variability contributing to the nondeterminism of degradation: (1) temporal variability; (2) unit-to-unite variability; and (3) measurement variability [28]. The temporal variability is referred to as the inherent stochastic characteristics of the standard Brownian motion. The measurement variability is usually considered while the degradation parameters were estimated. The unit-to-unite variability is usually modeled as random effects of the degradation parameter. It is very difficult to model the accelerated processes if the 
drift parameter $\lambda$ and the diffusion parameter $\sigma_{B}$ are both considered as the random parameters. So like Peng and Tseng [29], Si et al. [30, 31], and Tsai et al. [32], it is also assumed that different units have different drift parameters while all diffusion parameters have the same value under a certain stress. Then, we have $\eta \sim N\left(\mu_{\eta}, \sigma_{\eta}^{2}\right)$, where $\mu_{\eta}$ and $\sigma_{\eta}^{2}$ are the mean and variance of the parameter $\eta$, separately.

Considering the random effects and the effects of accelerated stresses on the drift parameter $\lambda$ and the diffusion parameter $\sigma_{B}$, we have the PDF and CDF of FHT under stress $S_{k}$ as

$$
\begin{aligned}
f_{S_{k}}(t) & =\frac{w}{\sqrt{2 \pi \omega^{3}\left(\kappa \varsigma_{k}+\sigma_{\eta}^{2} \varsigma_{k}^{2} \omega\right)}} \exp \left(-\frac{\left(w-\mu_{\eta} \varsigma_{k} \omega\right)^{2}}{2 \omega\left(\kappa \varsigma_{k}+\sigma_{\eta}^{2} \varsigma_{k}^{2} \omega\right)}\right) \frac{d \omega}{d t}, \\
F_{S_{k}}(t) & = \begin{cases}\Phi\left(\frac{\mu_{\eta} \varsigma_{k} \omega-w}{\sqrt{\omega\left(\kappa \varsigma_{k}+\sigma_{\eta}^{2} \varsigma_{k}^{2} \omega\right)}}\right)+\exp \left(\frac{2 \mu_{\eta} w}{\kappa}+\frac{2 \sigma_{\eta}^{2} w^{2}}{\kappa^{2}}\right) \cdot \Phi\left(-\frac{2 \sigma_{\eta}^{2} \varsigma_{k} w \omega+\kappa\left(\mu_{\eta} \varsigma_{k} \omega+w\right)}{\kappa \sqrt{\kappa \varsigma_{k} \omega+\sigma_{\eta}^{2} \varsigma_{k}^{2} \omega^{2}}}\right), & \text { if } t<\infty \\
\Phi\left(\frac{\mu_{\eta}}{\sigma_{\eta}}\right)+\exp \left(\frac{2 \mu_{\eta} w}{\kappa}+\frac{2 \sigma_{\eta}^{2} w^{2}}{\kappa^{2}}\right) \Phi\left(-\frac{2 \sigma_{\eta}^{2} w+\kappa \mu_{\eta}}{\kappa \sigma_{\eta}}\right), & \text { if } t \longrightarrow \infty,\end{cases}
\end{aligned}
$$

where $\Phi(\cdot)$ is the distribution function of the standard normal distribution.

When $\mu_{\eta} / \sigma_{\eta}$ approach infinity, the transformed FHT under stress $S_{k}$ is subject to inverse Gaussian (IG) distribution.

\section{Model the CSADP and SSADP with Random Effects}

As the most-used ADT, CSADT and SSADT have been widely researched. But the models are quite different while the drift parameter $\lambda$ and the diffusion parameter $\sigma_{B}$ all depend on stress variables, especially for SSADT.

3.1. Modeling the CSADP with Random Effects. Let $S_{1}$, $\ldots, S_{k}, \ldots, S_{K}$ denote $K$ stress level higher than normal stress level $S_{0}$ such that $S_{1}<\cdots<S_{K}$ and the subscript $k$ is corresponding to the $k$ th stress level, $k=1,2, \ldots, K$. Suppose that there are $N_{k}$ units of samples tested under a constant accelerated stress $S_{k}$ and each sample is measured $M_{k i}$ times at the $k$ th stress level, $i=1,2, \ldots, N_{k}$. The degradation at transformed time $\omega_{k i j}$ where $\omega_{k i j}=\Lambda\left(t_{k i j}\right)$ is $x_{k i j}, j=$ $1,2, \ldots, M_{k i}$. The degradation process can be formulated as

$$
x_{k i j}\left(\omega_{k i j} \mid S_{k}\right)=\eta_{i} \varsigma_{k} \omega_{k i j}+\sqrt{\kappa \varsigma_{k}} B\left(\omega_{k i j}\right) .
$$

3.2. Modeling the SSADP with Random Effects. Similarly, with CSADT, it was assumed that there are $K$ accelerated stress levels, $S_{1}<\cdots S_{k}<\cdots<S_{K}$, in the whole test. But the number of the samples is only $N$ which is different from $\sum_{k=1}^{K} N_{k}$ in CSADT that verifies the characteristic that SSADT needs fewer samples compared with CSADT [33]. Suppose that each sample is also measured $M_{k i}$ times at the $k$ th stress level, $i=1,2, \ldots, N, k=1,2, \ldots, K$. And $\tau_{k i}$ is the transformed time scale where $\tau_{k i}=\Lambda\left(t_{k i M_{k i}}\right)$ at which the stress changes from the $k$ th stress level to the $(k+1)$ th stress level of the $i$ th sample, $k=1,2, \ldots, K-1$. Besides, $\tau_{i 0}$ equals
0 and $\tau_{i K}$ is the transformed end time of the $K$-step stress accelerated test of the $i$ th sample.

The degradation process for CSADT is the same as SSADT under accelerated stress $S_{1}$.

$$
x_{1 i j}=\eta_{i} \varsigma_{1} \omega_{1 i j}+\sqrt{\kappa \varsigma_{1}} B\left(\omega_{1 i j}\right), \quad 0 \leq \omega_{1 i j} \leq \tau_{1 i} .
$$

Raise the accelerated stress up to $S_{2}$ at transformed time point $\tau_{1 i}$ for the $i$ th product. Then the degradation is driven by the accelerated stress $S_{2}$ and the corresponding parameters can be obtained by (9) are (10) where the drift parameter and the diffusion parameter are equal to $\eta_{i} \varsigma_{2}$ and $\kappa \varsigma_{2}$, separately. Thus, the degradation consisted of the degradation driven by $S_{1}$ and the degradation driven by $S_{2}$.

$$
\begin{aligned}
x_{2 i j}= & \eta_{i} \varsigma_{1} \tau_{i 1}+\sqrt{\kappa \varsigma_{1}} B\left(\tau_{i 1}\right)+\eta_{i} \varsigma_{2}\left(\omega_{i 2 j}-\tau_{i 1}\right) \\
& +\sqrt{\kappa \varsigma_{2}} B\left(\omega_{i 2 j}-\tau_{i 1}\right) .
\end{aligned}
$$

Similarly, the accelerated stress is turning up to $S_{3}$ at transformed time point $\tau_{2 i}$ and the degradation can be written as

$$
\begin{aligned}
x_{3 i j}= & \sum_{n=1}^{2}\left[\eta_{i} \varsigma_{n}\left(\tau_{i n}-\tau_{i(n-1)}\right)+\sqrt{\kappa \varsigma_{n}} B\left(\tau_{i n}-\tau_{i(n-1)}\right)\right] \\
& +\eta_{i} \varsigma_{3}\left(\omega_{i 3 j}-\tau_{i 2}\right)+\sqrt{\kappa \varsigma_{3}} B\left(\omega_{i 3 j}-\tau_{i 2}\right) .
\end{aligned}
$$


According to the analysis, the degradation process of SSADT can be formulated as

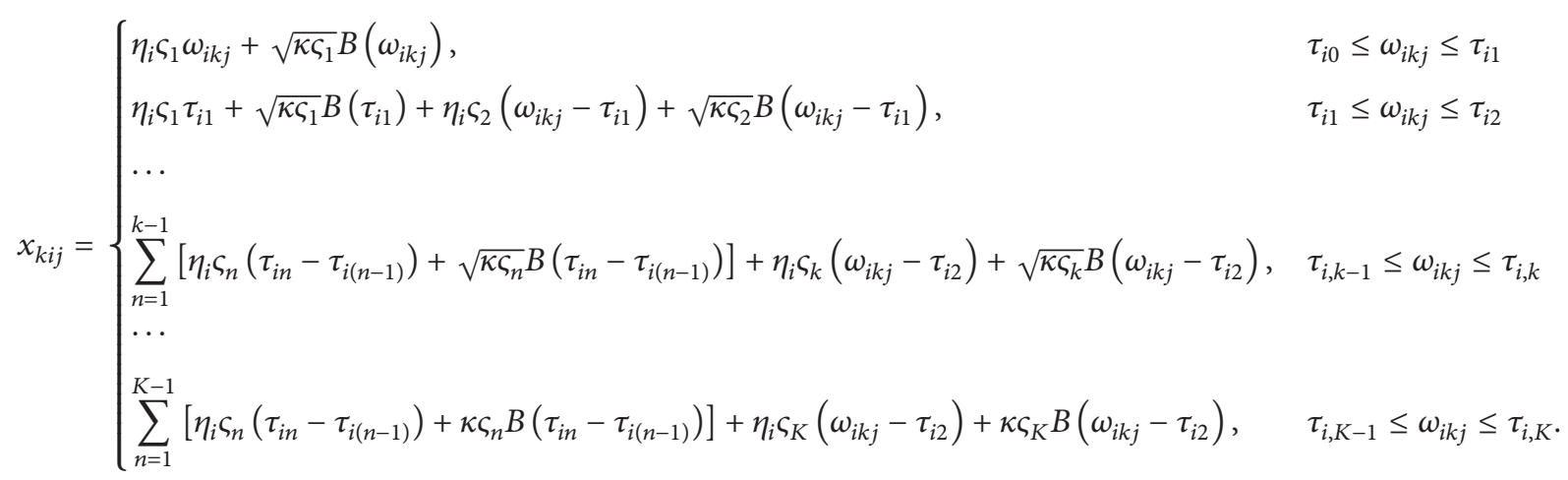

\section{Parameter Estimation}

In real applications, it is inevitable that some measurement errors may be introduced during the observation process [34]. When a measurement is taken, the observed degradation is shown as follows for both CSADT and SSADT:

$$
\begin{aligned}
& y_{k i j}\left(\omega_{k i j} \mid S_{k}\right)=x_{k i j}\left(\omega_{k i j} \mid S_{k}\right)+\varepsilon_{k i j} \\
& \quad k=1, \ldots, K ; i=1, \ldots, N_{k} ; j=1, \ldots, M_{k i},
\end{aligned}
$$

where the measurement errors $\varepsilon_{k i j}$ are assumed to be i.i.d. realizations of $\varepsilon_{k i j} \sim N\left(0, \sigma_{\varepsilon}^{2}\right)$ and mutually independent of $x_{k i j}[7,34]$ and $N_{k}$ is equal to $N$ for all of the accelerated stresses of SSADT.

The unknown parameters in the models are $\Theta=$ $\left\{\mu_{\eta}, \sigma_{\eta}^{2}, \kappa, \beta, \sigma_{\varepsilon}^{2}\right\}$. There are two methods to deal with the unknown parameters with each considering one of the two main properties of Brownian motion which could be characterized as follows [35]:

(P1) The increment $\Delta x(\omega)=x(\omega+\Delta \omega)-x(\omega)$ is independent of the degradation $x(\omega)$, which means that if $0 \leq \omega_{1} \leq \omega_{2} \leq \omega_{3} \leq \omega_{4}$, then $x\left(\omega_{2}\right)-$ $x\left(\omega_{1}\right)$ and $x\left(\omega_{4}\right)-x\left(\omega_{3}\right)$ are independent random variables. Meanwhile, the increment $\Delta x(\omega)$ follows a normal distribution where the mean equals $\lambda \Delta \omega$ and the variance is $\sigma_{B}^{2} \Delta \omega$.

(P2) According to (P1), the degradation $x(\omega)$ follows a normal distribution with the mean $\lambda \omega$ and the variance $\sigma_{B}^{2} \omega$.

Even though our models concern the unit-to-unite variability, the essence of the Wiener process remains the same. Owing to space constraints, this paper deals with the unknown parameter based on property (P2) in the case of CSADT and property (P1) in the case of SSADT.

4.1. Parameter Estimation of CSADT. Specify $T_{k i}=\left(T_{k i 1}\right.$, $\left.\ldots, T_{k i M_{k j}}\right)^{\prime}, T_{k i j}=\omega_{k i j}, Y_{k i}=\left(Y_{k i 1}, \ldots, Y_{k i M_{k i}}\right)^{\prime}, Y_{k}=$ $\left(Y_{k 1}, \ldots, Y_{k N_{k}}\right)$, and $Y=\left(Y_{1}, \ldots, Y_{K}\right)$ for $k=1, \ldots, K, i=$
$1, \ldots, N_{k}$, and $j=1, \ldots, M_{k i}$. According to property $(\mathrm{P} 1)$ and independent assumption of Brownian motion, $Y_{k i}$ follows a multivariate normal distribution with mean and variance:

$$
\begin{aligned}
& \tilde{\mu}_{k i}=\mu_{\eta} \varsigma_{k} T_{k i}, \\
& \Sigma_{k i}=\sigma_{\eta}^{2} \widetilde{\Sigma}_{k i},
\end{aligned}
$$

where

$$
\begin{aligned}
\widetilde{\Sigma}_{k i} & =\widetilde{\Omega}_{k i}+\varsigma_{k}^{2} T_{k i} T_{k i}^{T}, \\
Q_{k i} & =\left[\begin{array}{cccc}
\omega_{k i 1} & \omega_{k i 1} & \cdots & \omega_{k i 1} \\
\omega_{k i 1} & \omega_{k i 2} & \cdots & \omega_{k i 2} \\
\vdots & \vdots & \ddots & \vdots \\
\omega_{k i 1} & \omega_{k i 2} & \cdots & \omega_{k i M_{k i}}
\end{array}\right], \\
\Omega_{k i} & =\widetilde{\kappa} \varsigma_{k} Q_{k i}+\widetilde{\sigma}_{\varepsilon}^{2} I_{k i}
\end{aligned}
$$

and $I_{k i}$ is an identified matrix of order $M_{k i}$.

The log-likelihood function of unknown parameters $\widetilde{\Theta}=$ $\left\{\mu_{\eta}, \sigma_{\eta}^{2}, \widetilde{\kappa}, \beta, \tilde{\sigma}_{\varepsilon}^{2}\right\}$ is

$$
\begin{aligned}
& \ln L(\widetilde{\Theta} \mid Y) \\
& =-\frac{1}{2} \ln (2 \pi) \sum_{k=1}^{K} \sum_{i=1}^{N_{k}} M_{i k}-\frac{1}{2} \ln \left(\sigma_{\eta}^{2}\right) \sum_{k=1}^{K} \sum_{i=1}^{N_{k}} M_{k i} \\
& \quad-\frac{1}{2} \sum_{k=1}^{K} \sum_{i=1}^{N_{k}}\left|\widetilde{\Sigma}_{k i}\right| \\
& \quad-\frac{1}{2 \sigma_{\eta}^{2}} \sum_{k=1}^{K} \sum_{i=1}^{N_{k}}\left(y_{k i}-\mu_{\eta} \varsigma_{k} T_{k i}\right)^{\prime} \widetilde{\Sigma}_{k i}^{-1}\left(y_{k i}-\mu_{\eta} \varsigma_{k} T_{k i}\right) .
\end{aligned}
$$


Taking the first partial derivatives of the log-likelihood function with respect to $\mu_{\eta}, \sigma_{\eta}^{2}$ yields

$$
\begin{aligned}
& \frac{\partial \ln L(\widetilde{\Theta} \mid Y)}{\partial \mu_{\eta}} \\
& =\frac{1}{\sigma_{\eta}^{2}}\left(\sum_{k=1}^{K} \sum_{i=1}^{N_{k}} \varsigma_{k} T_{k i}^{\prime} \widetilde{\Sigma}_{k i}^{-1} y_{k i}-\mu_{\eta} \sum_{k=1}^{K} \sum_{i=1}^{N_{k}} \varsigma_{k}^{2} T_{k i}^{\prime} \widetilde{\Sigma}_{k i}^{-1} T_{k i}\right) \\
& \frac{\partial \ln L(\widetilde{\Theta} \mid Y)}{\partial \sigma_{\eta}^{2}}=-\frac{1}{2 \sigma_{\eta}^{2}} \sum_{k=1}^{K} \sum_{i=1}^{N_{k}} M_{k i}+\frac{1}{2\left(\sigma_{\eta}^{2}\right)^{2}} \\
& \cdot \sum_{k=1}^{K} \sum_{i=1}^{N_{k}}\left(y_{k i}-\mu_{\eta} \varsigma_{k} T_{k i}\right)^{\prime} \widetilde{\Sigma}_{k i}^{-1}\left(y_{k i}-\mu_{\eta} \varsigma_{k} T_{k i}\right) .
\end{aligned}
$$

For the special value of $\left(\widetilde{\kappa}, \beta, \widetilde{\sigma}_{\varepsilon}^{2}\right)$, setting the derivation of $\partial \ln L(\widetilde{\Theta} \mid Y) / \partial \mu_{\eta}, \partial \ln L(\widetilde{\Theta} \mid Y) / \partial \sigma_{\eta}^{2}$ to zero, the MLE for $\mu_{\eta}, \sigma_{\eta}^{2}$ can be expressed as

$$
\begin{aligned}
\widehat{\mu}_{\eta} & =\frac{\sum_{k=1}^{K} \sum_{i=1}^{N_{k}} \varsigma_{k} T_{k i}^{\prime} \widetilde{\Sigma}_{k i}^{-1} y_{k i}}{\sum_{k=1}^{K} \sum_{i=1}^{N_{k}} \varsigma_{k}^{2} T_{k i}^{\prime} \widetilde{\Sigma}_{k i}^{-1} T_{k i}}, \\
\widehat{\sigma}_{\eta}^{2} & =\frac{1}{\sum_{k=1}^{K} \sum_{i=1}^{N_{k}} M_{k i}} \\
& \cdot \sum_{k=1}^{K} \sum_{i=1}^{N_{k}}\left(y_{k i}-\widehat{\mu}_{\eta} \varsigma_{k} T_{k i}\right)^{\prime} \widetilde{\Sigma}_{k i}^{-1}\left(y_{k i}-\widehat{\mu}_{\eta} \varsigma_{k} T_{k i}\right) .
\end{aligned}
$$

Substituting (24) into (22) and simplifying, the profile log-likelihood function can be written as

$$
\begin{aligned}
\ln L(\widetilde{\Theta} \mid Y)= & -\frac{1}{2}(\ln (2 \pi)+1) \sum_{k=1}^{K} \sum_{i=1}^{N_{k}} M_{i k} \\
& -\frac{1}{2} \ln \left(\widehat{\sigma}_{\eta}^{2}\right) \sum_{k=1}^{K} \sum_{i=1}^{N_{k}} M_{k i} \\
& -\frac{1}{2} \sum_{k=1}^{K} \sum_{i=1}^{N_{k}}\left|\widetilde{\Sigma}_{k i}\right| .
\end{aligned}
$$

The MLE of $\tilde{\kappa}, \beta$, and $\widetilde{\sigma}_{\varepsilon}^{2}$ can be obtained by maximizing the profile log-likelihood function in (25) through a threedimensional search. Then, substitute them into (24); we can obtain MLE for $\mu_{\eta}$ and $\sigma_{\eta}^{2}$.

The value of $\widehat{\kappa}$ and $\widehat{\sigma}_{\varepsilon}^{2}$ can be obtained by the following equations:

$$
\begin{gathered}
\widehat{\kappa}=\widehat{\widetilde{\kappa}} \cdot \widehat{\sigma}_{\eta}^{2}, \\
\widehat{\sigma}_{\varepsilon}^{2}=\widehat{\widetilde{\sigma}}_{\varepsilon}^{2} \cdot \widehat{\sigma}_{\eta}^{2} .
\end{gathered}
$$

4.2. Parameter Estimation of SSADT. The degradation process of SSADT shown as (18) is relatively complicated. Thus we introduce the accelerated factor first for the sake of simplicity. The variable $\zeta_{k}$ is represented by the product of $\zeta_{1}$ and the accelerated factor $\alpha_{k, 1}$.

$$
\varsigma_{k}=\varsigma_{1} \alpha_{k, 1}
$$

The SSADP can be rewritten as

$$
\begin{aligned}
& x_{k i j} \\
& = \begin{cases}\eta_{i} \varsigma_{1} \omega_{1 i j}+\sqrt{\kappa \varsigma_{1}} B\left(\omega_{12 j}\right), & \tau_{i 0} \leq \omega_{i k j} \leq \tau_{i 1} \\
\eta_{i} \varsigma_{1}\left(\tau_{1 i}+\alpha_{2,1}\left(\omega_{2 i j}-\tau_{1 i}\right)\right)+\sqrt{\kappa \varsigma_{1}} B\left(\tau_{1 i}+\alpha_{2,1}\left(\omega_{2 i j}-\tau_{1 i}\right)\right) \\
\cdots & \tau_{1 i} \leq \omega_{2 i j} \leq \tau_{2 i} \\
\eta_{i} \varsigma_{1}\left(\sum_{n=1}^{k-1} \alpha_{n, 1}\left(\tau_{n i}-\tau_{(n-1) i}\right)+\alpha_{2,1}\left(\omega_{k i j}-\tau_{(k-1) i}\right)\right)+\sqrt{\kappa \varsigma_{1}} B\left(\sum_{n=1}^{k-1} \alpha_{n, 1}\left(\tau_{n i}-\tau_{(n-1) i}\right)+\alpha_{2,1}\left(\omega_{k i j}-\tau_{(k-1) i}\right)\right), & \tau_{(k-1) i} \leq \omega_{k i j} \leq \tau_{k i} \\
\ldots & \\
\eta_{i} \varsigma_{1}\left(\sum_{n=1}^{K-1} \alpha_{n, 1}\left(\tau_{n i}-\tau_{(n-1) i}\right)+\alpha_{2,1}\left(\omega_{K i j}-\tau_{(K-1) i}\right)\right)+\sqrt{\kappa \varsigma_{1}} B\left(\sum_{n=1}^{K-1} \alpha_{n, 1}\left(\tau_{n i}-\tau_{(n-1) i}\right)+\alpha_{2,1}\left(\omega_{K i j}-\tau_{(K-1) i}\right)\right), & \tau_{(K-1) i} \leq \omega_{K i j} \leq \tau_{K i} .\end{cases}
\end{aligned}
$$

Specify $\chi_{i k j}=\sum_{n=1}^{k-1} \alpha_{n, 1}\left(\tau_{i n}-\tau_{i(n-1)}\right)+\alpha_{2,1}\left(\omega_{i k j}-\tau_{i(k-1)}\right)$; then the SSADP can be expressed a general formula as follows:

$$
\begin{aligned}
& x_{k i j}=\eta_{i} \varsigma_{1} \chi_{k i j}+\sqrt{\kappa \varsigma_{1}} B\left(\chi_{k i j}\right), \\
& \tau_{(k-1) i} \leq \omega_{k i j} \geq \omega_{k i} \\
& \quad k=1, \ldots, K ; i=1, \ldots, N ; j=1, \ldots, M_{k i} .
\end{aligned}
$$

The foregoing transformation is equivalent to converting the degradation driven by stress $S_{k}$ to the degradation under stress $S_{1}$ in physics and the converted equivalent time is $\chi_{k i j}$. At the moment, the matrix of degradation and equivalent transformed time are still bidimensional. Because of the continuity of the degradation process, the matrix can be written as column vector with $M_{i}$ element where $M_{i}$ equals the sum of $M_{i k}, k=1, \ldots, K$, and the subscript can be written 
as $m$ for the $i$ th product, $m=1, \ldots, M_{i}$. Then the observed degradation can be expressed as

$$
\begin{aligned}
& y_{i m}=x_{i m}+\varepsilon_{i m}, \\
& x_{i m}=\eta_{i} \varsigma_{1} \chi_{i m}+\kappa \varsigma_{1} B\left(\chi_{i m}\right),
\end{aligned}
$$

$$
1 \leq m \leq \sum_{k=1}^{K} M_{i k},
$$

Define $\Delta \chi_{i 1}=\chi_{i 1}, \Delta \chi_{i m}=\chi_{i m}-\chi_{i(m-1)}, \Delta \chi_{i}=$ $\left(\Delta \chi_{i 1}, \ldots, \Delta \chi_{i M_{i}}\right)^{\prime}, R_{i}=\Delta \chi_{i}$, and $\Delta y_{i m}=y_{i m}-y_{i(m-1)}$ for $i=1, \ldots, N, m=1, \ldots, M_{i}$. Then $\Delta y_{i}=\left(\Delta y_{i 1}, \ldots, \Delta y_{i M_{i}}\right)^{\prime}$ follows a multivariate normal distribution $N\left(\mu_{\eta} \varsigma_{1} R_{i}, \Pi_{i}\right)$, where

$$
\begin{aligned}
\Pi_{i} & =W_{i}+\sigma_{\eta}^{2} \varsigma_{1}^{2} R_{i} R_{i}^{\prime}, \\
\left(W_{p, q}\right)_{i} & =\operatorname{cov}\left(\Delta y_{i p}, \Delta y_{i q}\right) \\
& = \begin{cases}\kappa \varsigma_{1} R_{i p}+\sigma_{\varepsilon}^{2}, & p=q=1 ; \\
\kappa \varsigma_{1} R_{i p}+2 \sigma_{\varepsilon}^{2}, & p=q>1 ; \\
-\sigma_{\varepsilon}^{2}, & p=q+1 \text { or } p=q-1 ; \\
0, & \text { otherwise. }\end{cases}
\end{aligned}
$$

So the log-likelihood function can be expressed as

$$
\begin{aligned}
\ln L(\Theta \mid Y) \\
=-\frac{1}{2} \ln (2 \pi) \sum_{i=1}^{N} M_{i}-\frac{1}{2} \sum_{i=1}^{N} \ln \left(\left|\Pi_{i}\right|\right) \\
\quad-\frac{1}{2} \sum_{i=1}^{N}\left(\Delta y_{i}-\mu_{\eta} \varsigma_{1} R_{i}\right)^{\prime} \Pi_{i}^{-1}\left(\Delta y_{i}-\mu_{\eta} \varsigma_{1} R_{i}\right),
\end{aligned}
$$

where

$$
\begin{aligned}
& \left|\Pi_{i}\right|=\left|W_{i}\right|\left(1+\sigma_{\eta}^{2} \varsigma_{1}^{2} R_{i}^{\prime} W_{i}^{-1} R_{i}\right) \\
& \Pi_{i}^{-1}=W_{i}^{-1}-\frac{\sigma_{\eta}^{2} \varsigma_{1}^{2}}{1+\sigma_{\eta}^{2} \varsigma_{1}^{2} R_{i}^{\prime} W_{i}^{-1} R_{i}} W_{i}^{-1} R_{i} R_{i}^{\prime} W_{i}^{-1} .
\end{aligned}
$$

Taking the first partial derivatives of the log-likelihood function with respect to $\mu_{\eta}, \sigma_{\eta}^{2}$ yields

$$
\begin{aligned}
& \frac{\partial \ln L(\Theta \mid Y)}{\partial \mu_{\eta}}=\varsigma_{1} \sum_{i=1}^{N} R_{i}^{\prime} \Pi_{i}^{-1} \Delta y_{i}-\mu_{\eta} \varsigma_{1}^{2} \sum_{i=1}^{N} R_{i}^{\prime} \Pi_{i}^{-1} R_{i}, \\
& \frac{\partial \ln L(\Theta \mid Y)}{\partial \sigma_{\eta}^{2}}=-\sum_{i=1}^{N} \frac{\varsigma_{1}^{2} R_{i}^{\prime} W_{i}^{-1} R}{1+\sigma_{\eta}^{2} \varsigma_{1}^{2} R_{i}^{\prime} W_{i}^{-1} R_{i}} \\
& +\sum_{i=1}^{N} \frac{\varsigma_{1}^{2}\left(\Delta y_{i}-\mu_{\eta} \varsigma_{1} R_{i}\right)^{\prime} W_{i}^{-1} R_{i} R_{i}^{\prime} W_{i}^{-1}\left(\Delta y_{i}-\mu_{\eta} \varsigma_{1} R_{i}\right)}{\left(1+\sigma_{\eta}^{2} \varsigma_{1}^{2} R_{i}^{\prime} W_{i}^{-1} R\right)^{2}}
\end{aligned}
$$

For the special value of $\left(\kappa, \beta, \sigma_{\varepsilon}^{2}\right)$, setting the derivation of $\partial \ln L(\Theta \mid Y) / \partial \mu_{\eta}$ to zero, the restricted MLE for $\mu_{\eta}$ can be expressed as

$$
\widehat{\mu}_{\eta}=\frac{\sum_{i=1}^{N} R_{i}^{\prime} \Pi_{i}^{-1} \Delta y_{i}}{\varsigma_{1} \sum_{i=1}^{N} R_{i}^{\prime} \Pi_{i}^{-1} R_{i}} .
$$

Similarly, set the derivation of $\partial \ln L(\Theta \mid Y) / \partial \mu_{\eta}$ to zero and the results of the MLE for $\sigma_{\eta}^{2}$ will be discussed on two cases.

Case 1. It was assumed that the number of the measurements and the measurement points of each sample are the same for all of the samples under all of the accelerated stress. That is to say, the subscript of $R_{i}, \Pi_{i}$, and $W_{i}$ can be removed.

Thus, the restricted MLE for $\mu_{\eta}$ can be expressed as

$$
\widehat{\mu}_{\eta}=\frac{\sum_{i=1}^{N} R^{\prime} \Pi_{i}^{-1} \Delta y_{i}}{\varsigma_{1} N R^{\prime} \Pi_{i}^{-1} R} .
$$

The first partial derivatives of the log-likelihood function to $\sigma_{\eta}^{2}$ can be rewritten as

$$
\begin{aligned}
& \frac{\partial \ln L(\Theta \mid Y)}{\partial \sigma_{\eta}^{2}}=-N \frac{\varsigma_{1}^{2} R^{\prime} W^{-1} R}{1+\sigma_{\eta}^{2} \varsigma_{1}^{2} R^{\prime} W^{-1} R} \\
& +\frac{\varsigma_{1}^{2}}{\left(1+\sigma_{\eta}^{2} \varsigma_{1}^{2} R^{\prime} W^{-1} R\right)^{2}} \\
& \quad \sum_{i=1}^{N}\left(\Delta y_{i}-\mu_{\eta} \varsigma_{1} R\right) W^{-1} R R^{\prime} W^{-1}\left(\Delta y_{i}-\mu_{\eta} \varsigma_{1} R\right)^{\prime} .
\end{aligned}
$$

For the special value of $\left(\kappa, \beta, \sigma_{\varepsilon}^{2}\right)$, setting the derivation of $\partial \ln L(\Theta \mid Y) / \partial \sigma_{\eta}^{2}$ to zero, the result of the MLE for $\sigma_{\eta}^{2}$ can be expressed as

$$
\begin{aligned}
\widehat{\sigma}_{\eta}^{2} & \\
= & \frac{\sum_{i=1}^{N}\left(\Delta y_{i}-\mu_{\eta} \varsigma_{1} R\right) W^{-1} R R^{\prime} W^{-1}\left(\Delta y_{i}-\mu_{\eta} \varsigma_{1} R\right)^{\prime}}{N \varsigma_{1}^{2}\left(R^{\prime} W^{-1} R\right)^{2}} \\
& -\frac{1}{\varsigma_{1}^{2} R^{\prime} W^{-1} R} .
\end{aligned}
$$

Substituting (37), (39), into (32) and simplifying, the profile log-likelihood function can be written as

$$
\begin{array}{r}
\ln L\left(\kappa, \beta, \sigma_{\varepsilon}^{2} \mid \widehat{\mu}_{\eta}, \widehat{\sigma}_{\eta}^{2}, Y\right)=-\frac{M N}{2} \ln (2 \pi)-\frac{N}{2}-\frac{N}{2} \\
\cdot \ln (|W|) \\
-\frac{1}{2}\left\{\sum_{i=1}^{N} \Delta y_{i}^{\prime} W^{-1} \Delta y_{i}-\frac{\sum_{i=1}^{N}\left(R^{\prime} W^{-1} \Delta y_{i}\right)^{2}}{R^{\prime} W^{-1} R}\right\}-\frac{1}{2} \\
\cdot \ln \left\{\frac{\sum_{i=1}^{N}\left(R^{\prime} W^{-1} \Delta y_{i}\right)^{2}}{N R^{\prime} W^{-1} R}-\frac{\sum_{i=1}^{N}\left(R^{\prime} W^{-1} \Delta y_{i}\right)^{2}}{N^{2} R^{\prime} W^{-1} R}\right\},
\end{array}
$$


where $M$ is the measurement time point of each sample which is the same for all of the samples based on the assumption of Case 1.

The MLE of $\kappa, \beta$, and $\sigma_{\varepsilon}^{2}$ can be obtained by maximizing the profile log-likelihood function in (40) through a threedimensional search. Then, substitute them into (37) and (39); we can obtain the MLE for $\mu_{\eta}$ and $\sigma_{\eta}^{2}$.

Case 2. The number of the measurements and the measurement points of each sample are different for all of the samples under all of the accelerated stress. In this case, the first partial derivatives of the log-likelihood function to $\sigma_{\eta}^{2}$ are shown as in (35). There may be no analytical form by setting the partial derivatives to zero. The evaluation of $\mu_{\eta}$ can be expressed as (36). Then, the profile log-likelihood function of $\sigma_{\eta}^{2}, \kappa, \beta, \sigma_{\varepsilon}^{2}$ can be written as

$$
\begin{aligned}
\ln L\left(\kappa, \beta, \sigma_{\varepsilon}, \sigma_{\eta}^{2} \mid \widehat{\mu}_{\eta}, Y\right) \\
=-\frac{1}{2} \ln (2 \pi) \sum_{i=1}^{N} M_{i}-\frac{1}{2} \sum_{i=1}^{N} \ln \left(\left|\Pi_{i}\right|\right) \\
\quad-\sum_{i=1}^{N}\left(\Delta y_{i}-\widehat{\mu}_{\eta} \varsigma_{1} R_{i}\right) \Pi_{i}^{-1}\left(\Delta y_{i}-\widehat{\mu}_{\eta} \varsigma_{1} R_{i}\right)^{\prime} .
\end{aligned}
$$

The MLE of $\sigma_{\eta}^{2}, \kappa, \beta$, and $\sigma_{\varepsilon}^{2}$ can be obtained by maximizing the profile log-likelihood function in (41) through a four-dimensional search. Then, substitute them into (36); we can obtain the MLE for $\mu_{\eta}$.

It is not to say that we can only use degradation for CSADT and increment for SSADT but just make an introduction to both of the two methods in the limited space. In addition, we could verify the results by comparing the estimation calculated by the two methods to avoid computation errors.

\section{Simulation Data Analysis}

In order to validate the model described before and the parameter estimation methods, simulation test was conducted.

5.1. The Simulation Method of CSADT Data. The parameters $\Theta=\left\{\mu_{\eta}, \sigma_{\eta}^{2}, \kappa, \beta, \sigma_{\varepsilon}^{2}\right\}$ should be given before the simulation and simulation process is shown as in the following steps.

Step 1 . Set $k=1$.

Step 2. Generate $N_{i}$ random numbers with subscripts $i, i=$ $1,2, \ldots, N_{k}$, subject to normal distribution with mean $\mu_{\eta}$ and variance $\sigma_{\eta}^{2}$.

Step 3. Let $l=0$.

Step 4. Calculate $X_{k i(l+1)}$ using the Euler approximation [36]

$$
X_{k i(l+1)}=X_{k i l}+\eta_{i} \varsigma_{k} \omega(\Delta t)+\kappa \varsigma_{k} \sqrt{\omega(\Delta t)} \Psi \text {, }
$$

where $\Psi$ is the random number according to the standard normal distribution and $\Delta t$ is the step size in simulation and $X_{k i 0}=0$. As such, set $l=l+1$, and return to Step 4 to get the degradation path until $l=L_{k i}+1$, where $L_{k i}$ is the simulation steps for $i$ th under stress $S_{k}$.

Step 5. Set $k=k+1$ and return to Step 2 until $k=K+1$.

Step 6. Extract the degradation based on the predefined measurement time point $t_{k i j}$ where $k=1,2, \ldots, K, i=$ $1,2, \ldots, N_{k}$, and $j=1,2, \ldots, M_{k i}$, from the data set $X_{k i l}$ and get the data set of degradation $X_{k i j}$.

Step 7. Simulate the measured degradation $Y_{k i j}=X_{k i j}+\varepsilon_{k i j}$, where $\varepsilon_{k i j} \sim N\left(0, \sigma_{\varepsilon}^{2}\right)$.

5.2. The Simulation Method of SSADT Data. There are some differences in the simulation process for SSADT compared with CSADT. The simulation process is shown as follows.

Step 1. Generate $N$ random numbers with subscripts $i, i=$ $1,2, \ldots, N$, subject to normal distribution with mean $\mu_{\eta}$ and variance $\sigma_{\eta}^{2}$.

Step 2. Let $k=1$.

Step 3. Set $l=1$.

Step 4. Calculate $X_{k i(l+1)}$ :

$$
X_{k i(l+1)}=X_{k i l}+\eta_{i} \varsigma_{k} \omega(\Delta t)+\kappa \varsigma_{k} \sqrt{\omega(\Delta t)} \Psi,
$$

where $\Psi$ is the random number according to the standard normal distribution and $\Delta t$ is the step size in simulation and $X_{k i 0}=0, X_{k i 0}=X_{(k-1) i L_{(k-1)}}(k>1)$. As such, set $l=l+1$, and return to Step 4 to get the degradation path until $l=L_{k i}+1$.

Step 5. Set $k=k+1$ and return to Step 2 until $k=K+1$.

Step 6. Extract the degradation based on the predefined measurement time point $t_{k i j}$ where $k=1,2, \ldots, K, i=$ $1,2, \ldots, N$, and $j=1,2, \ldots, M_{i k}$, from the data set $X_{k i l}$ and get the data set of degradation $X_{k i j}$.

Step 7. Simulate the measured degradation $Y_{k i j}=X_{k i j}+\varepsilon_{k i j}$, where $\varepsilon_{k i j} \sim N\left(0, \sigma_{\varepsilon}^{2}\right)$.

5.3. The Analysis of the Simulated SSADT Data. We just give the analysis of simulated SSADT data here because we would give a case study of the CSADT later. It was assumed that the accelerated stress is temperature and the simulation test contains 4 stresses which are $50^{\circ} \mathrm{C}, 60^{\circ} \mathrm{C}, 70^{\circ} \mathrm{C}$, and $80^{\circ} \mathrm{C}$. For simplicity, the transformed time function is set as $\Lambda(t)=t$ and 20 degradation paths are generated. Then, the parameters for degradation process are assumed as $\Theta=\{5,4,0.25,-3000,0.01\}$. Moreover, the time interval measurement is $25 \mathrm{~h}$ for each sample of the whole simulation test with 40 measurement time points. The degradation data are depicted as in Figure 1. 
TABLE 2: The parameters of three degradation models with the SSADT simulated degradation data.

\begin{tabular}{lccccccrrr}
\hline & $\mu_{\eta}$ & $\sigma_{\eta}^{2}$ & $\kappa$ & $\sigma_{B}^{2}$ & $\beta$ & $\sigma_{\varepsilon}^{2}$ & $\log$-LF & AIC & RE \\
\hline Truth value & 5 & 1 & 0.25 & - & -3000 & 0.01 & - & - \\
$M_{0}$ & 5.01 & 1.39 & 0.28 & - & -2975.61 & 0.0078 & 1422.28 & -2834.56 & 0.8402 \\
$M_{1}$ & 5.12 & 1.75 & - & $1.2907 * 10^{-4}$ & -2980.81 & 0.0129 & 1122.89 & -2235.77 & 5.6271 \\
$M_{2}$ & 6.20 & - & - & $1.5213 * 10^{-4}$ & -3051.75 & $1.3789 * 10^{-9}$ & 1093.61 & -2177.21 & 7.8049 \\
\hline
\end{tabular}

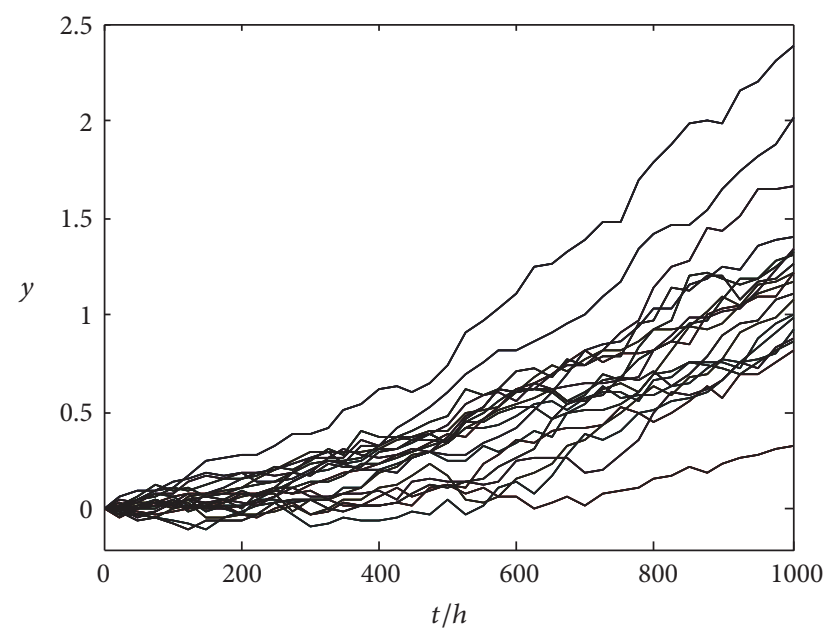

Figure 1: The simulation degradation paths of SSADT.

For simplicity, the degradation model for SSADT proposed in this paper is referred to as $M_{0}$, the model presented by Tang et al. [17] as $M_{1}$, and the stochastic SSADT model described in reference [13] as $M_{2}$. The estimation results of the unknown parameters, the log-likelihood function value, and the Akaike information criterion (AIC) [37], which is evaluated by (44), are summarized in Table 2 . As we can see from Table 2, our model clearly outperforms model in terms of the log-LF and AIC. The estimates of $\mu_{\eta}, \sigma_{\eta}^{2}$, and $\sigma_{\varepsilon}^{2}$ are more close to the truth values compared with $M_{1}$ and $M_{2}$. Meanwhile, the diffusion-related variables include parameters $\kappa$ and $\beta$ and the diffusion parameter under the four stresses can be obtained by (10) which is $\left(2.8377 * 10^{-5}\right.$, $\left.4.8534 * 10^{-5}, 7.8244 * 10^{-5}, 1.2001 * 10^{-4}\right)$ in $M_{1}$ and $\left(2.2334 * 10^{-5}, 3.9912 * 10^{-5}, 6.4597 * 10^{-5}, 9.9423 * 10^{-5}\right)$ for the true model. Moreover, taking $S_{1}$ as a reference, a criterion formulizing the relative error (RE) is shown as (45). It is clear that $M_{0}$ has the smallest RE from Table 2.

$$
\begin{aligned}
\operatorname{AIC}(N)= & -2 \ln L(\Theta)+2 N, \\
\operatorname{RE}= & \left|\frac{\mu_{\eta}-\widehat{\mu}_{\eta}}{\widehat{\mu}_{\eta}}\right|+\left|\frac{\sigma_{\eta}^{2}-\widehat{\sigma}_{\eta}^{2}}{\widehat{\sigma}_{\eta}^{2}}\right|+\left|\frac{\left(\sigma_{B}^{2}\right)_{1}-\left(\widehat{\sigma}_{B}^{2}\right)_{1}}{\left(\widehat{\sigma}_{B}^{2}\right)_{1}}\right| \\
& +\left|\frac{\beta-\widehat{\beta}}{\widehat{\beta}}\right|+\left|\frac{\sigma_{\varepsilon}^{2}-\widehat{\sigma}_{\varepsilon}^{2}}{\widehat{\sigma}_{\varepsilon}^{2}}\right| .
\end{aligned}
$$

It is assumed that the failure threshold $w$ is equal to 5 and the normal stress is $25^{\circ} \mathrm{C}$. The PDF and CDF under normal stress are shown as in Figure 2. The phenomenon can be observed where the PDF and CDF of our model most nearly approached the real model. By contrasting $M_{1}$ with $M_{2}$, it can be concluded that the neglect of random effects would result in sharper PDF which is consistent with the result in reference [17]. And it is noteworthy that the 0.5 quantiles of the failure life are almost equal from the CDF of the three models. But there is a greater difference between the real model and $M_{1}$ while the indicator is taken as mean time to failure, as well as the difference between the real model and $M_{2}$. So the correlation between the stress and the diffusion parameter should not be neglected. Next, we are going to prove the superiority of our model in practical application.

\section{Case Application}

The CSADT model with covariates and random effects is verified by the accelerated degradation data of carbon-film resistors whose raw data set is explicitly given in Table C.3 of Meeker and Escobar [38]. There were 29 samples and three accelerated temperature stresses in the whole test, where nine samples were observed at $83^{\circ} \mathrm{C}$; the remaining two stresses, $133^{\circ} \mathrm{C}$ and $173^{\circ} \mathrm{C}$, had 10 samples for each stress. All of the samples were observed at the same time points with $t_{0}=0$, $t_{1}=452, t_{2}=1030, t_{3}=4341$, and $t_{4}=8084$ (in hours). Similar to [39], it was assumed that the standard operating temperature was $50^{\circ} \mathrm{C}$ and the threshold value for percent increase in resistance was taken to be $C=12 \%$. It is reasonable and computationally easier to use the ratios in resistance to the initial value for each rather than the percent increase [9]. For ease of calculation, the logarithm of the ratio was taken as the degradation here. That is, the value 0.28 of the percent increase was changed to $2.796 E-3$ and so on and the transformed threshold value $w$ was 0.1133 . The degradation paths of all the sample were as shown in Figure 3. It can be seen that the degradation of the samples uniformly showed a nonlinear characteristic especially at the beginning of the ADT. Thus, the degradation is modeled with the time scale transformed Wiener process as (1) with $\omega=\Lambda(t)=t^{b}$.

Similarly, the proposed degradation model for SSADT in this paper is referred to as $M_{0}$, the model presented by Tang et al. [17] as $M_{1}$, and the model described in reference [9] as $M_{3}$. The estimation results of the unknown parameters, the loglikelihood function value, and the AIC are shown in Table 3. The estimated parameters $b$ are approximately equal for the three models. Take $b=0.50$ as an example to show the time 
TABLE 3: The parameters of three degradation models with the SSADT simulated degradation data.

\begin{tabular}{ccccccccccc}
\hline & $\mu_{\eta}$ & $\sigma_{\eta}^{2}$ & $\kappa$ & $\sigma_{B}^{2}$ & $\beta$ & $\sigma_{\varepsilon}^{2}$ & $b$ & Log-LF & AIC & MTTF \\
\hline$M_{0}$ & 8.23 & 2.07 & $1.08 E-02$ & - & -4202.82 & $1.82 E-20$ & 0.50 & 518.48 & -1024.97 & $3.80 E+07$ \\
$M_{1}$ & 14.71 & 4.33 & - & $4.13 E-07$ & -4586.56 & $5.06 E-20$ & 0.53 & 486.37 & -960.73 & $5.15 E+07$ \\
$M_{2}$ & 11.11 & - & - & $6.76 E-07$ & -4479.82 & $4.11 E-18$ & 0.53 & 468.37 & -926.74 & $4.06 E+07$ \\
\hline
\end{tabular}
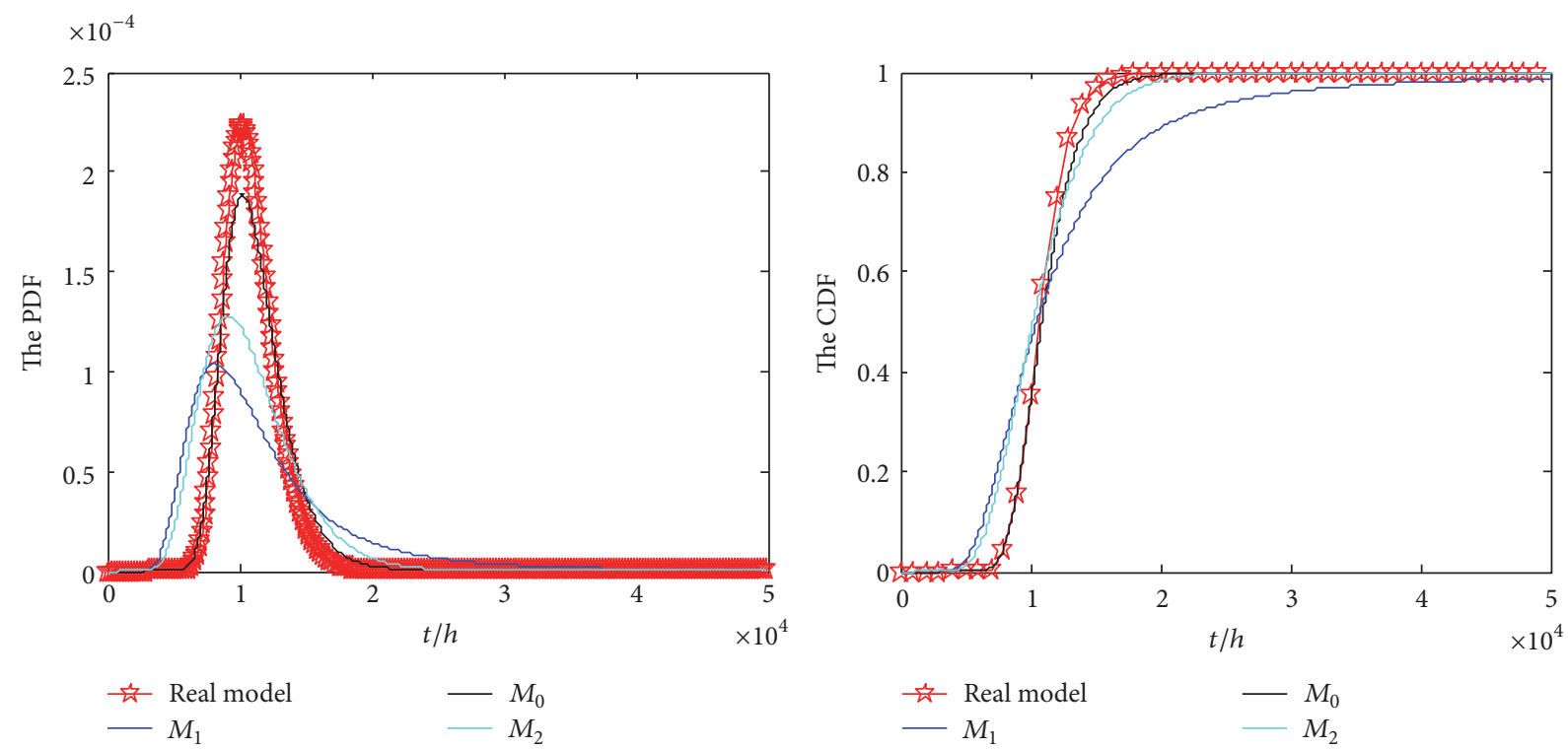

FIGURE 2: The comparison of the PDF and CDF of the three models for simulated SSADT data.

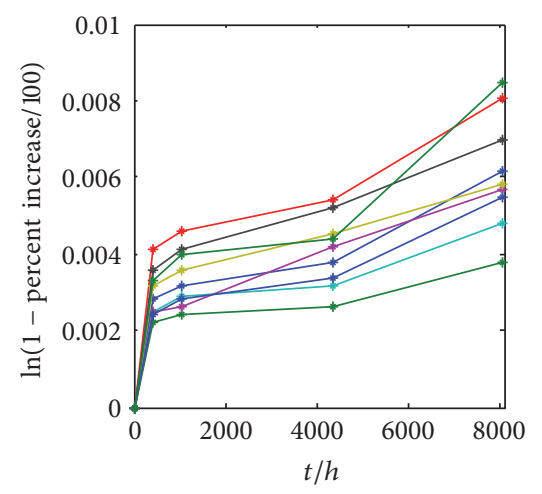

(a) $S=83^{\circ} \mathrm{C}$

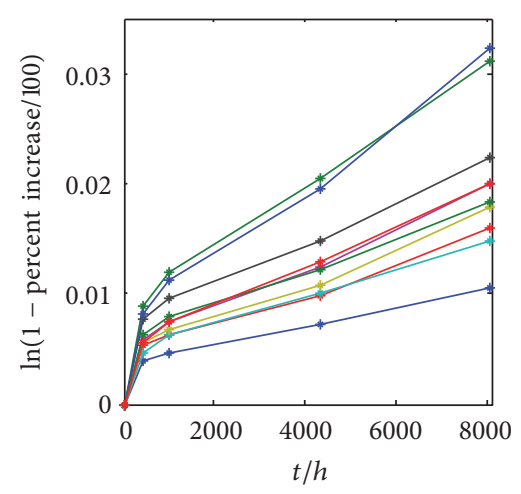

(b) $S=133^{\circ} \mathrm{C}$

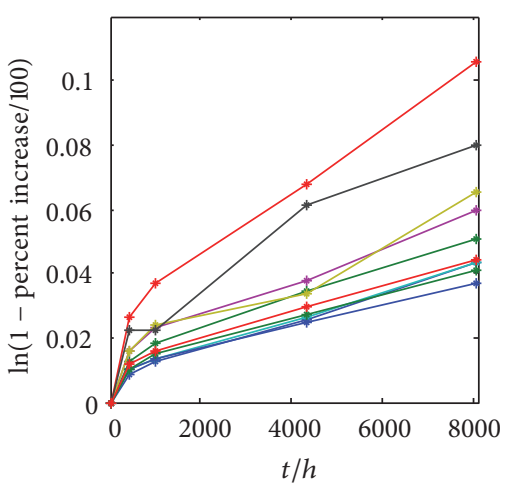

(c) $S=173^{\circ} \mathrm{C}$

FIgURE 3: The degradation paths of carbon-film resistors.

scale transformed degradation paths as in Figure 4 which are approximately straight lines compared with Figure 3.

Our model has the largest log-LF and smallest AIC compared with $M_{1}$ and $M_{2}$. The result proves that our model is more suitable for the degradation data. Besides, $M_{1}$ has the larger log-LF and smaller AIC compared with $M_{2}$. So the random effects could not be neglected. It can be observed that the variance of the drift parameter is slightly larger which may be owing to the fixed value of diffusion parameter in $M_{1}$.

The PDF and CDF under the standard operating temperature are as shown in Figure 5. The time corresponding to the peak values of PDF were as follows: $2.676 * 10^{7}, 1.049 * 10^{7}$, and $5.028 * 10^{6}$ for $M_{0}$ to $M_{2}$. But the MTTF of $M_{0}$ is minimal from Table 3. And the uncertainty in the estimated PDFs of the lifetimes under $M_{0}$ is smallest compared to $M_{1}$ and $M_{2}$, as seen in Figure 5. When time $t$ approaches positive infinity, the CDF of our model which is calculated by (13) tends illimitably to 1 and the CDF of $M_{1}$ is roughly equal to 0.9997 . It follows that our model could do well in distinguishing the difference from random effects and the effect of covariates. Another thing which is worth noting is that the measurement error is quite tiny in the case but it is just an individual phenomenon. The measurement error should also be considered in practical application. 


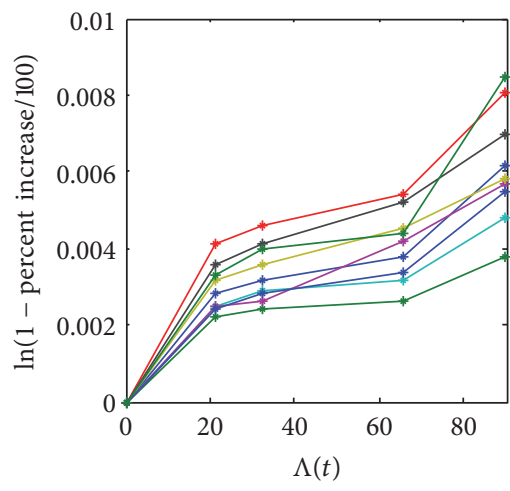

(a) $S=83^{\circ} \mathrm{C}$

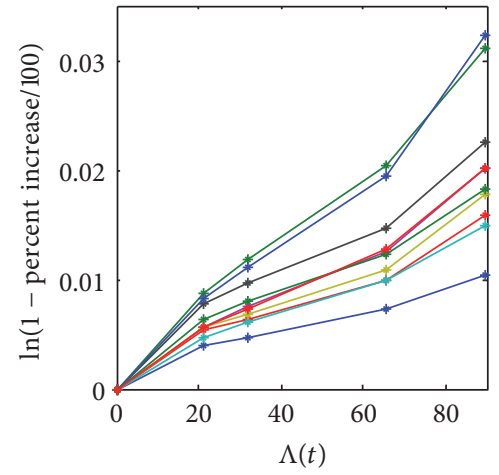

(b) $S=133^{\circ} \mathrm{C}$

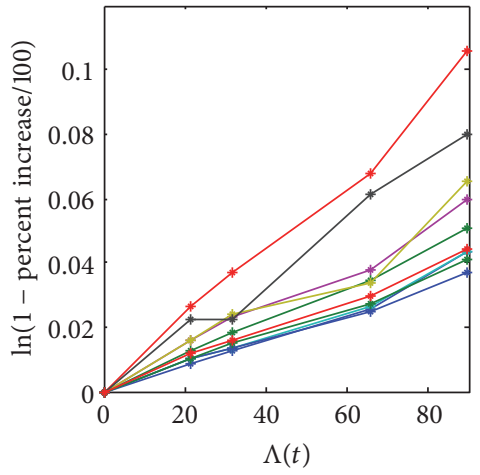

(c) $S=173^{\circ} \mathrm{C}$

FIGURE 4: The degradation paths of carbon-film resistors under transformed time scale.
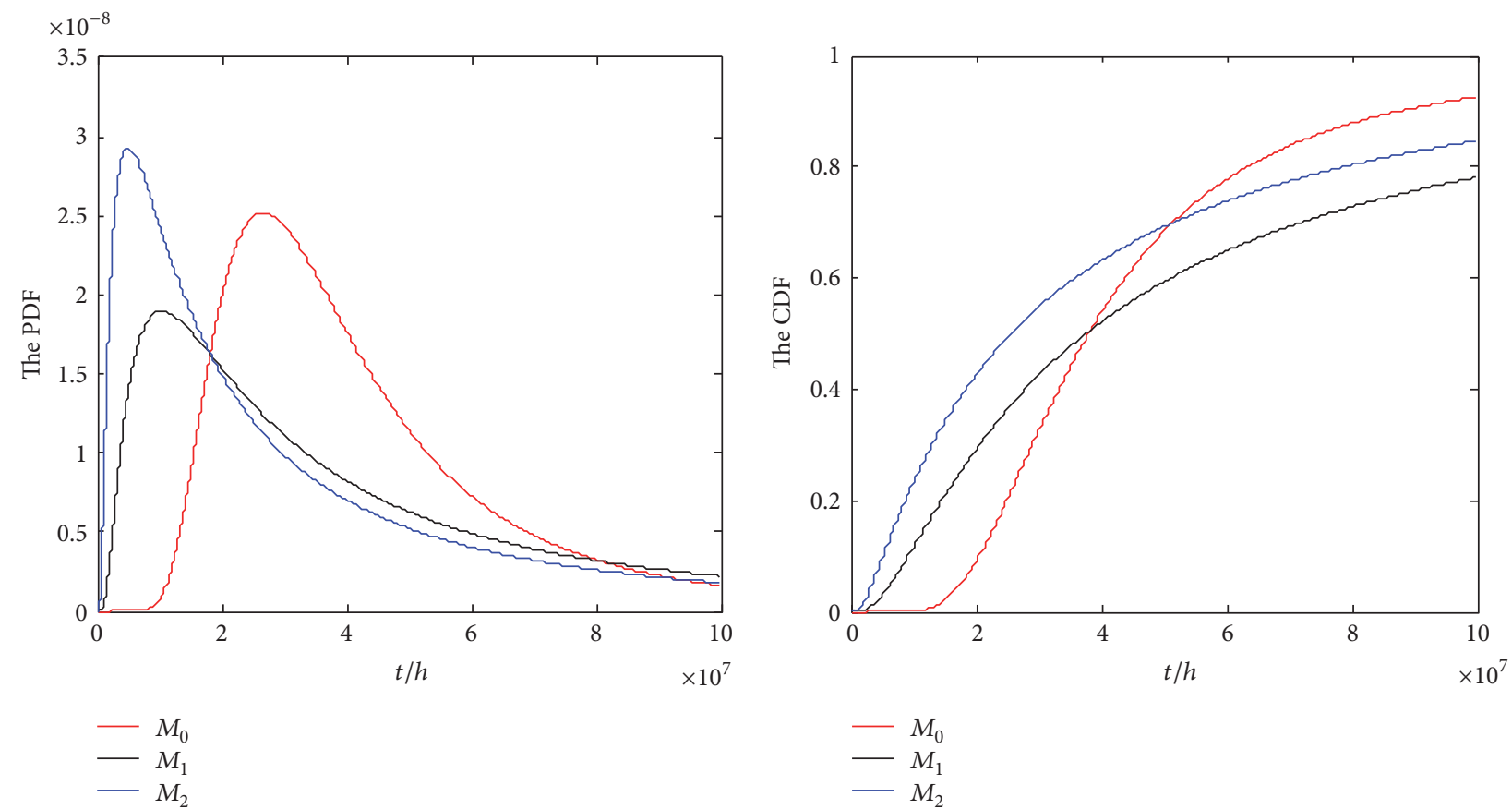

FIGURE 5: The comparison of the PDF and CDF of the three models for real CSADT data.

\section{Conclusion}

In this paper, the degradation models based on nonlinear Wiener process are established for both constant stress accelerated degradation data and step stress accelerated degradation data. Before the establishment, the relationship between the drift parameter and stress variables is derived based on the invariance principle of failure mechanism and Nelson assumption, so is the relationship between the diffusion parameter and stress variables. It is concluded that the ratio of drift parameters under two stresses is a constant which is irrelevant to the testing time and depends only on the two stresses, as long as the ratio of diffusion parameters is equal to the ratio of drift parameters. And the ratio is defined as accelerated factor. Besides, the random effects are also taken into consideration where the drift parameter is assumed to be normally distributed and the diffusion parameter is same for all of the samples under a certain stress. Then the PDF and $\mathrm{CDF}$ of the FHT are deduced considering random effects.

Because of the dependency between the diffusion parameter and stress variables, the degradation process is quite different, either for CSADT or for SSADT. The CSADP and SSADP with random effects are modeled. Moreover, the unknown parameters are solved by MLE based on the two properties of Wiener process. At the end of the paper, the simulated data of SSADT and the CSADT data of carbon-film resistors are both analyzed to verify the proposed model. It is concluded that the model has the biggest log-LF and the smallest AIC compared with the two other models.

The innovation of this paper lies in the following: First, the random effects are considered under the new relationship between the diffusion parameter and accelerated stresses. 
Second, the degradation process was modeled for both CSADT and SSADT. Thirdly, the unknown parameters were estimated based on the two properties of Wiener process and the result of the MLE for $\sigma_{\eta}^{2}$ is discussed on two cases. Fourth, the measurement error of the degradation data is also valued.

However, we have only considered the random effects of the drift parameter in this paper due to the complexity of the computation. A further research may consider the random effects of the diffusion parameter into the model. At the same time, the study of the paper may provide new ideas for the relativity analysis between the parameters of other stochastic process and stress variables.

\section{Competing Interests}

The authors declare that there is no conflict of interests regarding the publication of this paper.

\section{Acknowledgments}

This research was supported by Jiangsu Province Graduate Student Scientific Research Innovation Project of China (Project KYLX15_0330). The help is gratefully acknowledged.

\section{References}

[1] H.-F. Yu, "Optimal classification of highly-reliable products whose degradation paths satisfy Wiener processes," Engineering Optimization, vol. 35, no. 3, pp. 313-324, 2003.

[2] X. Wang, N. Balakrishnan, and B. Guo, "Residual life estimation based on a generalized Wiener degradation process," Reliability Engineering and System Safety, vol. 124, pp. 13-23, 2014.

[3] Y. Ren, Q. Feng, T. Ye, and B. Sun, "A novel model of reliability assessment for circular electrical connectors," IEEE Transactions on Components, Packaging and Manufacturing Technology, vol. 5, no. 6, pp. 755-761, 2015.

[4] Z.-S. Ye and M. Xie, "Stochastic modelling and analysis of degradation for highly reliable products," Applied Stochastic Models in Business and Industry, vol. 31, no. 1, pp. 16-32, 2015.

[5] H. Lim and B.-J. Yum, "Optimal design of accelerated degradation tests based on Wiener process models," Journal of Applied Statistics, vol. 38, no. 2, pp. 309-325, 2011.

[6] C. J. Lu and W. Q. Meeker, "Using degradation measures to estimate a time-to-failure distribution," Technometrics, vol. 35, no. 2, pp. 161-174, 1993.

[7] G. A. Whitmore, "Estimating degradation by a wiener diffusion process subject to measurement error," Lifetime Data Analysis, vol. 1, no. 3, pp. 307-319, 1995.

[8] Z. Pan and N. Balakrishnan, "Reliability modeling of degradation of products with multiple performance characteristics based on gamma processes," Reliability Engineering \& System Safety, vol. 96, no. 8, pp. 949-957, 2011.

[9] C. Park and W. J. Padgett, "Accelerated degradation models for failure based on geometric Brownian motion and gamma processes," Lifetime Data Analysis, vol. 11, no. 4, pp. 511-527, 2005.

[10] Z.-S. Ye and N. Chen, "The inverse Gaussian process as a degradation model," Technometrics, vol. 56, no. 3, pp. 302-311, 2014.
[11] A. Onar and W. J. Padgett, "Accelerated test models with the inverse Gaussian distribution," Journal of Statistical Planning and Inference, vol. 89, no. 1-2, pp. 119-133, 2000.

[12] T.-R. Tsai, Y. L. Lio, and N. Jiang, "Optimal decisions on the accelerated degradation test plan under the Wiener process," Quality Technology and Quantitative Management, vol. 11, no. 4, pp. 461-470, 2014.

[13] C.-M. Liao and S.-T. Tseng, "Optimal design for step-stress accelerated degradation tests," IEEE Transactions on Reliability, vol. 55, no. 1, pp. 59-66, 2006.

[14] L. C. Tang, G. Yang, and M. Xie, "Planning of step-stress accelerated degradation test," in Proceedings of the Annual Reliability and Maintainability Symposium - Proceedings: International Symposium on Product Quality and Integrity, pp. 287-292, January 2004.

[15] C.-H. Hu, M.-Y. Lee, and J. Tang, "Optimum step-stress accelerated degradation test for Wiener degradation process under constraints," European Journal of Operational Research, vol. 241, no. 2, pp. 412-421, 2015.

[16] S. Tang, "Step stress accelerated degradation process modeling and remaining useful life estimation," Journal of Mechanical Engineering, vol. 50, no. 16, p. 33, 2014.

[17] S. Tang, X. Guo, C. Yu, H. Xue, and Z. Zhou, "Accelerated degradation tests modeling based on the nonlinear wiener process with random effects," Mathematical Problems in Engineering, vol. 2014, Article ID 560726, 11 pages, 2014.

[18] L. Wang, R. Pan, X. Li, and T. Jiang, "A Bayesian reliability evaluation method with integrated accelerated degradation testing and field information," Reliability Engineering \& System Safety, vol. 112, pp. 38-47, 2013.

[19] Z.-S. Ye, N. Chen, and Y. Shen, "A new class of Wiener process models for degradation analysis," Reliability Engineering \& System Safety, vol. 139, pp. 58-67, 2015.

[20] G. A. Whitmore and F. Schenkelberg, "Modelling accelerated degradation data using wiener diffusion with a time scale transformation," Lifetime Data Analysis, vol. 3, no. 1, pp. 27-45, 1997.

[21] K. A. Doksum and A. Hoyland, "Models for variable-stress accelerated life testing experiments based on Wiener processes and the inverse Gaussian distribution," Technometrics, vol. 34, no. 1, pp. 74-82, 1992.

[22] H. Liao and E. A. Elsayed, "Reliability inference for field conditions from accelerated degradation testing," Naval Research Logistics, vol. 53, no. 6, pp. 576-587, 2006.

[23] H.-W. Wang, T.-X. Xu, and W.-Y. Wang, "Remaining life prediction based on wiener processes with ADT prior information," Quality and Reliability Engineering International, vol. 32, no. 3, pp. 753-765, 2015.

[24] C.-Y. Peng, "Inverse Gaussian processes with random effects and explanatory variables for degradation data," Technometrics, vol. 57, no. 1, pp. 100-111, 2015.

[25] X. Pan, X. Huang, Y. Chen, Y. Wang, and R. Kang, "Connotation of failure mechanism consistency and identification method for accelerated testing," in Proceedings of the Prognostics and System Health Management Conference (PHM-Shenzhen '11), May 2011.

[26] W. B. Nelson, Accelerated Testing: Statistical Models, Test Plans, and Data Analysis, John Wiley \& Sons, 2009.

[27] Z.-S. Ye, Y. Hong, and Y. Xie, "How do heterogeneities in operating environments affect field failure predictions and test planning?" The Annals of Applied Statistics, vol. 7, no. 4, pp. 2249-2271, 2013. 
[28] Z.-X. Zhang, X.-S. Si, C.-H. Hu, Q. Zhang, T. Li, and C. Xu, "Planning repeated degradation testing for products with threesource variability," IEEE Transactions on Reliability, vol. 65, no. 2, pp. 640-647, 2016.

[29] C.-Y. Peng and S.-T. Tseng, "Mis-specification analysis of linear degradation models," IEEE Transactions on Reliability, vol. 58, no. 3, pp. 444-455, 2009.

[30] X.-S. Si, W. Wang, C.-H. Hu, D.-H. Zhou, and M. G. Pecht, "Remaining useful life estimation based on a nonlinear diffusion degradation process," IEEE Transactions on Reliability, vol. 61, no. 1, pp. 50-67, 2012.

[31] X.-S. Si, W. Wang, C.-H. Hu, M.-Y. Chen, and D.-H. Zhou, "A Wiener-process-based degradation model with a recursive filter algorithm for remaining useful life estimation," Mechanical Systems and Signal Processing, vol. 35, no. 1-2, pp. 219-237, 2013.

[32] C.-C. Tsai, S.-T. Tseng, and N. Balakrishnan, "Mis-specification analyses of gamma and Wiener degradation processes," Journal of Statistical Planning and Inference, vol. 141, no. 12, pp. 37253735, 2011.

[33] F. Haghighi and S. J. Bae, "Reliability estimation from linear degradation and failure time data with competing risks under a step-stress accelerated degradation test," IEEE Transactions on Reliability, vol. 64, no. 3, pp. 960-971, 2015.

[34] Z.-S. Ye, Y. Wang, K.-L. Tsui, and M. Pecht, "Degradation data analysis using wiener processes with measurement errors," IEEE Transactions on Reliability, vol. 62, no. 4, pp. 772-780, 2013.

[35] J. Huang, D. S. Golubović, S. Koh et al., "Degradation modeling of mid-power white-light LEDs by using Wiener process," Optics Express, vol. 23, no. 15, pp. A966-A978, 2015.

[36] A. Beskos, O. Papaspiliopoulos, G. O. Roberts, and P. Fearnhead, "Exact and computationally efficient likelihood-based estimation for discretely observed diffusion processes (with discussion)," Journal of the Royal Statistical Society Series B: Statistical Methodology, vol. 68, no. 3, pp. 333-382, 2006.

[37] J. Shang and J. E. Cavanaugh, "An assumption for the development of bootstrap variants of the Akaike information criterion in mixed models," Statistics and Probability Letters, vol. 78, no. 12, pp. 1422-1429, 2008.

[38] W. Q. Meeker and L. A. Escobar, Statistical Methods for Reliability Data, John Wiley \& Sons, New York, NY, USA, 1998.

[39] W. J. Padgett and M. A. Tomlinson, "Inference from accelerated degradation and failure data based on Gaussian process models," Lifetime Data Analysis, vol. 10, no. 2, pp. 191-206, 2004. 


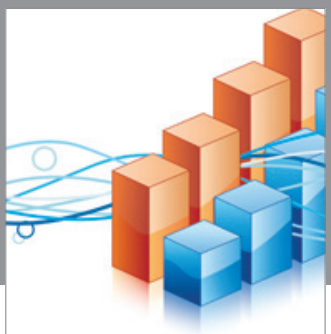

Advances in

Operations Research

vatem alat4

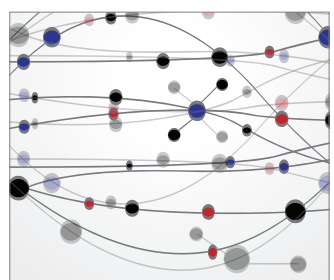

\section{The Scientific} World Journal
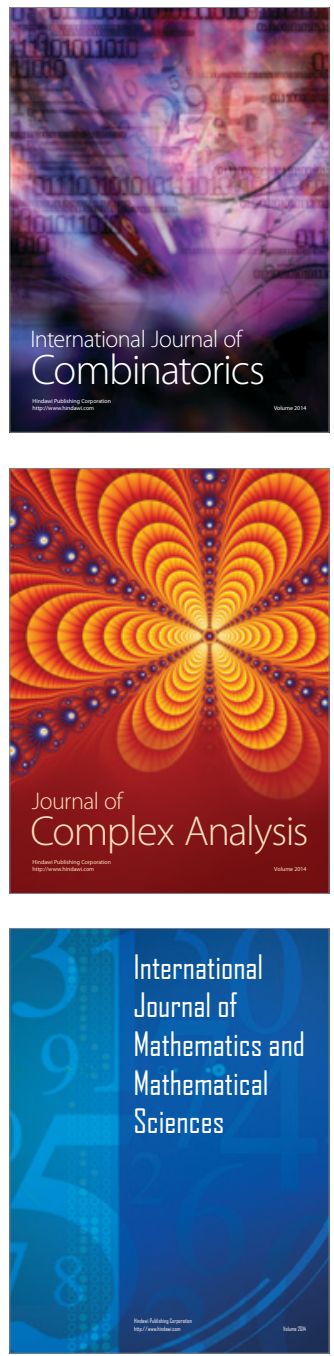
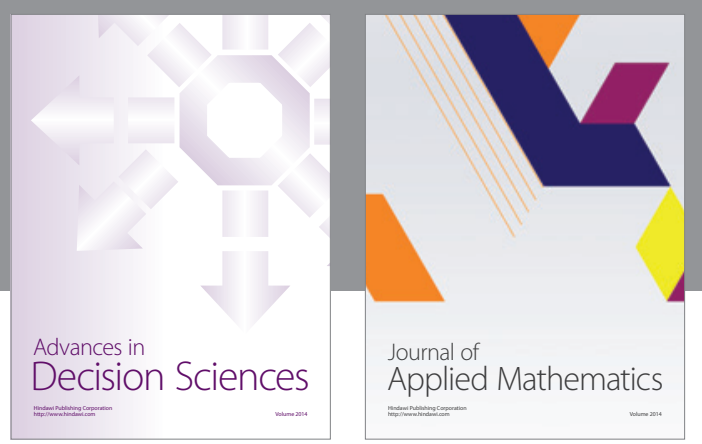

Algebra

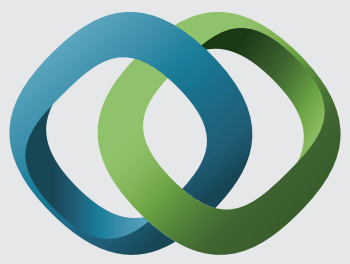

\section{Hindawi}

Submit your manuscripts at

http://www.hindawi.com
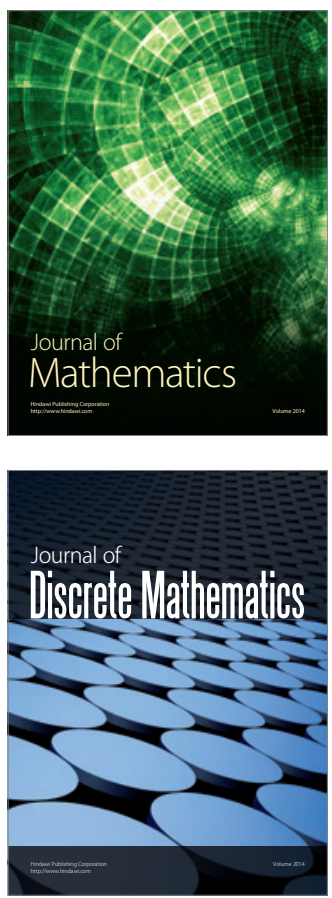

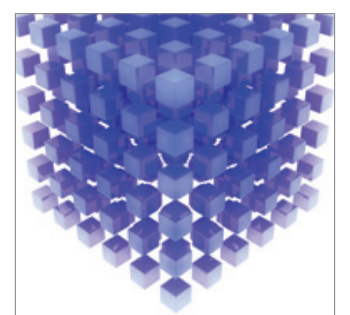

Mathematical Problems in Engineering
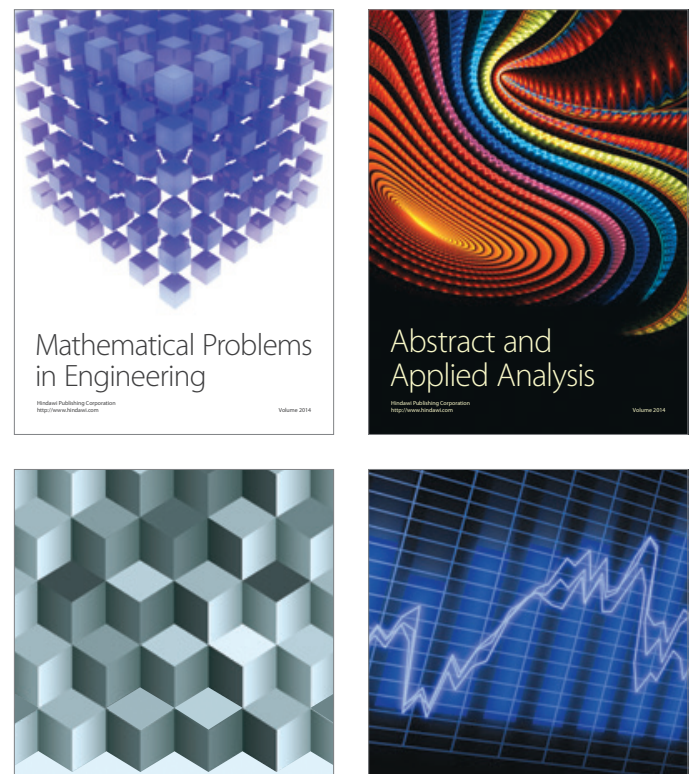

Journal of

Function Spaces

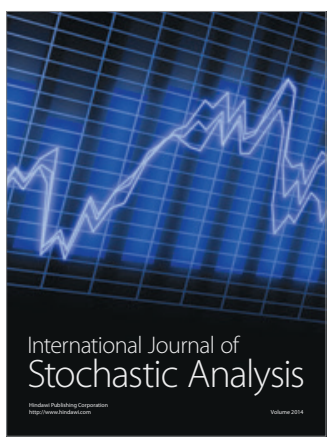

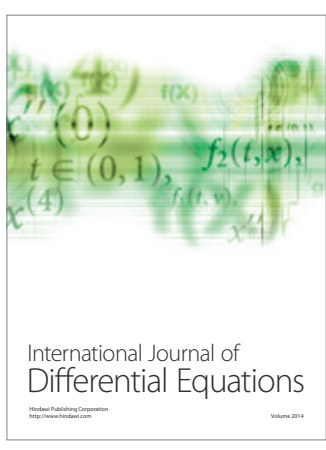
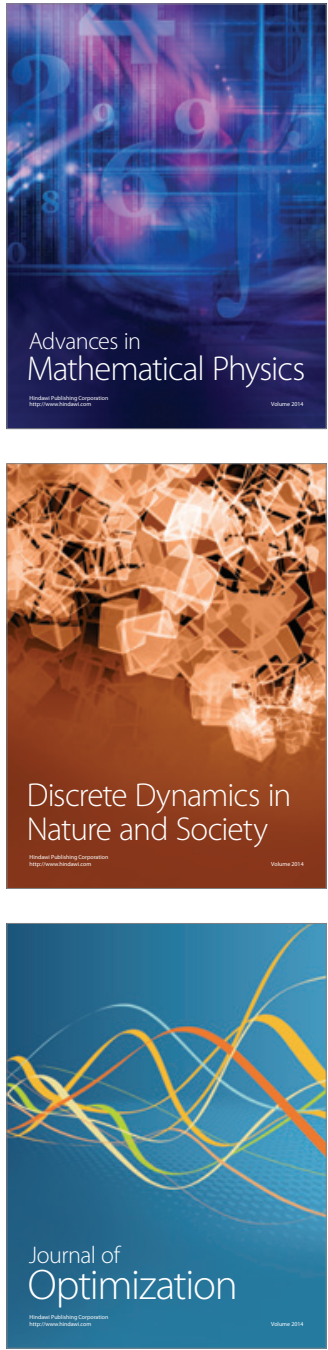